\title{
The Interstellar Medium in Nearby Galaxies
}

\author{
J. M. VAN DER HULST \\ Kapteyn Astronomical Institute, Groningen, The Netherlands
}

\begin{abstract}
Recent observations of the several phases of the interstellar medium in galaxies reveal a wide range of structures and physical properties. The HI structure in nearby galaxies is very filamentary with a large number of shells and filaments suggestive of a close interaction between the star formation in the disk and the surrounding interstellar medium.
\end{abstract}

Key words: Nearby Galaxies, Interstellar Medium, HI shells

\section{Introduction}

Our understanding of the structure and physical properties of the interstellar medium (ISM) in nearby galaxies has greatly advanced over the last years. Obvious reasons are that modern synthesis telescopes now allow imaging of the ISM in nearby galaxies on scales of a few hundred parsecs or less (about the size of large star forming regions) and that modern CCD technology allows imaging of faint emission line gas.

Good overviews of both observations and theory san be found in IAU Symposium 144 (1991) and the second Wyoming Conference (1990). Ideas concerning the energy balance in the ISM appear to converge to a common picture where star formation activity is a main source of energy input into the ISM via stellar winds and supernova events. This activity causes a variety of structures and properties, ranging from Heiles shells and worms to the diffuse ionized gas component which has a much larger scale height than the cool neutral gas layer.

Studies of our own Galaxy give the best information on the physical properties of the ISM. The limitation, however, is the edge-on vantage point for the observer and the limited range of star formation activity. If the properties of the ISM depend on factors such as star formation activity, metallicity, mass density etc. then one must observe the ISM in a number of galaxies in order to sample a large range of these. Studies of the ISM in other galaxies, therefore, are instrumental for furthering our understanding of the processes governing the state of the ISM.

In this paper I will address some of the more recent issues in ISM research in other galaxies with an emphasis on the small scale structure of the neutral component.

\section{Phases in the ISM}

The idea that the ISM in galaxies has several components with different temperatures and densities and in pressure equilibrium with one another has received wide acceptance since the pioneering work of Clark (1965) and Field, Goldsmith and Habing (1979). Currently more than the two phases proposed originally are favored. These are: a cold phase, mostly molecular gas, with temperatures between 20 and $80 \mathrm{~K}$, densities between 10 and $10^{3} \mathrm{~cm}^{-3}$ and a filling factor of $<0.1$; a cool phase, mostly HI, with temperatures between 80 and $300 \mathrm{~K}$, densities between 0.1 and $10 \mathrm{~cm}^{-3}$ and a filling factor of about 0.1 ; and a warm phase, mostly diffuse 
ionized hydrogen, with temperatures between $10^{3}$ and $10^{4} \mathrm{~K}$, densities of 0.05 to 0.1 $\mathrm{cm}^{-3}$ and a large filling factor of 0.2 to 0.7 . This latter phase is viewed as two components by Kulkarni and Heiles (1988) who distinguish between the warm neutral medium (WNM, HI at temperatures of a few $1000 \mathrm{~K}$, e.g. see Lockman 1984, 1991) and the warm ionised medium (WIM, e.g. see Reynolds 1984, 1991). In addition there is the extended, hot phase ( $\mathrm{T}>8000 \mathrm{~K})$, first hypothesized by Cox and Smith (1974) and described by McKee and Ostriker (1977) as the result of heating of a warm, partly ionized component by supernova events in the disk. They associated this phase with the hot "coronal" gas seen around the Galaxy as UV absorption lines against bright stars in the Magellanic Clouds (Savage and de Boer 1981, see also Savage 1991). The venting of hot gas into the corona or halo of a galaxy by supernovae in the disk is described in detail by the more recent chimney model of Norman and Ikeuchi (1989).

Each phase has its own distribution and small scale structure, though overlap in properties does exist. In the next sections the distribution and structure of the cold, cool and warm phase will be described separately. I will not discuss the hot phase.

\section{The Cold, Molecular Medium}

Surveys of CO in nearby spiral galaxies have shown that the cold, molecular component comprises a large fraction of the gas mass, in spite of the small filling factor. Most studies of the molecular gas in nearby galaxies to date have been made with single dishes giving angular resolutions between 20" and 50" (Young, 1990, Kenney 1990, Young and Scoville 1991). The molecular gas appears to have a more centrally concentrated distribution than the $\mathrm{HI}$ with the exception of early type galaxies with large bulges such as M 31, NGC 2841 and NGC 7331 (Stark 1979, Young and Scoville 1982). The molecular to atomic gas fraction decreases towards later Hubble types (Young and Knezek 1989), perhaps indicating a change in conditions for the formation of molecular gas and a change in metallicity. The scale height of the molecular gas layer in the Milky Way is about 80 parsecs (Blitz, 1991). Whether the scale height of the molecular gas in other galaxies is similar is not clear. García-Burillo et al. (1991) report CO detections in NGC 891 some 1 $\mathrm{kpc}$ above the plane using the 30-m IRAM dish. This is, however, not confirmed by Nobeyama data (Heiles, 1991).

The most complete study at higher angular resolution of $\mathrm{CO}$ in a nearby galaxy is that of M 51 (Rand and Kulkarni 1990, Rand 1990). In M 51 the dense CO is concentrated along the inside of the optical spiral arms, coincident with the dust lanes. This coincidence, combined with the observation that the HI peak column densities do not coincide with the $\mathrm{CO}$ peaks but instead are aligned with the downstream HII regions in the spiral arms, has lead Tilanus and Allen (1991) to suggest that the action of a spiral density wave causes star formation downstream of the spiral shock and that the newly born massive stars dissociate part of the molecular gas thus causing the observed enhancements in the HI. This displacement of the $\mathrm{HI}$ with respect to the dust and peak column densities in the $\mathrm{CO}$ is perhaps not a general phenomenon, as it is not clearly observed in NGC 6946 (Casoli et al. 1990, 
Tacconi and Xie 1990) and in M 83 (Lord and Kenney 1991).

A more recent analysis of the $\mathrm{CO}$ data of $\mathrm{M} 51$ in conjunction with $\mathrm{HI}, \mathrm{H \alpha}$, IRAS and B-band data (Rand et al. 1991) gives further support to the Allen and Tilanus picture. This study indicates in addition that a substantial fraction of the infrared emission arises from dust heated by photons which do not originate in massive star-forming regions. The same conclusion has been reached in studies of other nearby spiral galaxies: M 33 (Rice et al. 1990), M 101 (Beichman et al. 1987), NGC 4565, NGC 891 and NGC 5907 (Wainscoat et al. 1987). Studies of the Galaxy (Bloemen et al. 1990) and M 31 (Walterbos and Schwering 1987) suggest that most of the FIR emission arises from cirrus in these galaxies.

Detailed maps of nearby galaxies will be available soon from millimeter synthesis arrays such as BIMA and the OVRO array, and questions regarding the formation and destruction of molecular clouds, the size and stability of molecular cloud complexes, the effects of density waves etc. will be addressed with this new data.

\section{The Cool Neutral Medium}

\subsection{The temperature of The HI}

The cool, neutral component consists mostly of neutral atomic hydrogen at temperatures of one to a few hundred $\mathrm{K}$. The physical temperature of the gas has been estimated from emission - absorption studies of Galactic HI (Dickey et al. 1978, Payne et al. 1982, 1983, Colgan et al. 1988). Measurement of the absorption and the emission of the HI allows an estimate of the HI spin temperature under the assumption that the physical conditions and sampled line of sights are the same for the emitting and absorbing gas. The Galactic studies arrive at a range of temperatures between 50 and $150 \mathrm{~K}$. Similar studies in other galaxies are much more difficult because the number of suitably strong background sources diminishes rapidly with decreasing angular size. Yet such studies are possible in Local Group galaxies as the recent work on M 31 by Braun and Walterbos (1991, see also Braun 1991) indicates. A study of HI absorption and emission along several lines of sight in M31 indicates a temperature of $80-200 \mathrm{~K}$ for the cool gas in M31, possibly dependent on galactocentric distance within M31. The average spin temperature in M 31 appears somewhat higher than in the Galaxy which Braun and Walterbos ascribe to a higher pressure in the ISM. Braun and Walterbos propose a two component model fot the Galaxy and M 31: a warm phase with a scale height of 400 parsec and a cool phase with a scale height of 150 parsec. The temperatures of the warm and cool phase are about 175 and $8000 \mathrm{~K}$ respectively. These results are quite exciting but still limited by resolution and sensitivity. Future synthesis telescopes with large collecting areas are required to perform similar studies in nearby galaxies outside the Local Group. At the moment one is limited to probing the maximum brightness temperature of the $\mathrm{HI}$ emission. A study of eleven nearby galaxies is underway (Braun et al., see Braun 1991) and preliminary results indicate that maximum brightness temperatures vary from galaxy to galaxy and range from 30 to $230 \mathrm{~K}$ (Braun, priv. comm.). 


\subsection{The STRUCTURE OF ThE HI}

Heiles $(1979,1984)$ first pointed out the presence of shells and filaments in the HI in the Galaxy and tried to associate these with star formation in the disk. The situation in other galaxies has been reviewed recently by Braun (1991) and van der Hulst and Kamphuis (1991). Galaxies close enough that the HI can be resolved on spatial scales of 20 to 400 parsecs show a wealth of structure. Besides the general concentration of $\mathrm{HI}$ in spiral arms, recognized already 18 years ago when the first $\mathrm{HI}$ synthesis maps of nearby galaxies were made (van Allen et al. 1973), there appears to be a complex topology of filaments, perhaps sheets, shells and semi-shells, often termed holes in the past.

The first systematic attempts catalogue and study such holes were made in M 31 (Brinks and Bajaja 1986) and in M 33 (Deul and den Hartog 1990). The sizes of the majority of the holes identified in M 31 and M 33 are only a few hundred parsecs and the kinetic energies required to produce them are $10^{51-53}$ ergs. This implies that the collective effects of stellar winds and supernova explosions could very well produce these as indicated by the calculations of Mac Low and McCray (1988). Only the smaller ( $<300$ parsec) holes in M 31 appear to be associated with star forming regions (Brinks and Bajaja 1986), a notable exception being the hole associated with NGC 206 (Brinks, 1981). In M 31 Brinks et al. (1989) found some HI shells which do have $\mathrm{H} \alpha$ counterparts showing the same kinematic behaviour. In M 33 Deul and den Hartog (1990) applied several correlation techniques to investigate the spatial coincidence with $\mathrm{HII}$ regions and $\mathrm{OB}$-associations. Though some correlation was found the trend is not very strong.

The larger holes (up to a few $\mathrm{kpc}$ ) do not correlate very well with star forming regions, though some of these may just be interarm regions, classified as holes. A general problem is posed by the topology of the HI which appears to be very filamentary, possibly shaped by several generations of star formation, and could easily produce apparent holes just from projection effects. The energies required to produce the larger holes are $10^{53-54}$ ergs and are too large to be produced by the typical young stellar associations seen in $M 31$ and $M$ 33. An alternative explanation is the infall of large HI clouds (Tenorio-Tagle and Bodenheimer 1988, and references therein). A population of such clouds has, however, not yet been found in other galaxies, though the searches to date are sensitivity limited and could only have detected clouds more massive than $10^{6} \mathrm{M}_{\odot}$. An exception perhaps is the high velocity gas complex found in M 101 (van der Hulst and Sancisi 1988). Such a structure could eventually break up into smaller clouds which fall back into the disk and puncture holes in it.

Recent, sensitive observations of the large nearby galaxies M 101 and NGC 6946 by. Kamphuis have revealed the existence of a population of large shells and holes in these galaxies with sizes ranging from 0.5 to $5 \mathrm{kpc}$ and energies of $10^{53-54} \mathrm{ergs}$. Most of these supershells are not associated with star forming regions, though also here a very notable exception exists: the supershell near NGC 5462 in M 101 (Kamphuis et al. 1991). These shells are so large that they must have risen above the cool HI layer which has a scale height of only 100 - 200 parsec and probably represent examples of bubble breakouts as postulated by Mac Low et al. (1989). 
The size of the holes in the plane presumably is indicative for the scale height of the ISM component which prevents the bubbles from breaking out quickly, as the holes will stop expanding in the plane as soon as breakout occurs. The presence of magnetic fields could prevent early blow out as well as it tends to hold the gas down into the disk. Another aspect to take into account is that the larger holes in the plane may just result from several generations of star formation as new associations tend to form near old sites of star formation. Yet the observations of the Heiles shells and worms in our galaxy and the large holes in other objects such as M 31, M 33, M 101 and NGC 6946 do seem to support the idea of chimneys venting gas into the halo as proposed by Norman and Ikeuchi (1989).

\section{The Warm Ionized Medium}

The observations of Clark (1965) gave the first indication for the presence of the warm neutral component in the ISM. The study of Reynolds (1984) confirm its existence and show that this component is partly ionized. Deep $\mathrm{H} \alpha$ imaging of the edge-on galaxy NGC 891 by Rand et al. (1990) show that this diffuse ionised component extends to about $4 \mathrm{kpc}$ above the plane. The image also shows many filaments and extensions out to $>2 \mathrm{kpc}$ above the plane, reminiscent of the worms found in the Galaxy by Heiles (1984).

The $[\mathrm{SII}] / \mathrm{H} \alpha$ ratio of the diffuse gas above the plane is 0.3 to 0.5 , significantly higher than is observed in HII regions. Lasker $(1977,1979)$ found similarly high ratios in ionised. gas loops in the LMC. Such high ratios can result from photoionisation by a dilute photon field (Mathis 1986) which requires leakage of UV radiation out of the disk to large distances above the plane. The escape of ionizing photons through chimneys is discussed in detail by Norman (1991). Another edgeon galaxy observed in $\mathrm{H} \alpha$, NGC 4244, does not show an extended ionised gas disk (Walterbos 1991). NGC 4244, however, has a 10 times lower FIR luminosity than NGC 891, indicative of a much lower star formation rate. This may explain this difference in extent of the ionised gas layer. Recent observations of two more edgeon galaxies (Rand et al. 1991) futher support this picture. NGC 4631 with a FIR luminosity similar to NGC 891 has $\mathrm{H} \alpha$ emission at high $\mathrm{z}$, though patchy and not diffuse as in NGC 891. The other galaxy studied, NGC 4565 is weak in $\mathrm{H} \alpha$, shows no extended ionized gas disk and has a 6 times lower FIR luminosity than NGC 891.

Such a diffuse ionised gas component is also well known to exist in the Local Group galaxies M 31 (Walterbos 1990), M 33 (Courtèz et al. 1988), and the Magellanic Clouds (Davies et al. 1976, Meaburn 1980). The topology of this ionised gas component is complex, with many filaments, loops and bubbles, in addition to diffuse gas halos around the HII regions. Hunter and Gallagher (1989) have drawn attention to such a topology in Irregular galaxies and termed it froth.

\section{Acknowledgements}

I thank Jurjen Kamphuis and Robert Braun for letting me use unpublished material. I am very grateful to Jurjen Kamphuis and Richard Rand for reading an earlier version of this manuscript. 


\section{References}

Allen, R. J., Goss, W. M. and van Woerden, H. 1973 Astron. Astrophys 29, 447.

Blitz, L. 1990, in The Interstellar Disk-Halo Connection in Galaxies,

IAU Symp. 144, ed. J. B. G. M. Bloemen, (Kluwer Acad. Publ.) 41.

Beichman, C., Boulanger, F., Rice, W. and Lonsdale Persson, C. J. 1987, in Star Formation in Galaxies, NASA Publ. nr. CP 2466, ed. C. J. Lonsdale Persson, p. 297.

Bloemen, H., Deul, E., R. and Thaddeus, P. 1990 Astron. Astrophys, 233, 437.

Braun, R. 1990, in The Interstellar Disk-Halo Connection in Galaxies,

IAU Symp. 144, ed. J. B. G. M. Bloemen, (Kluwer Acad. Publ.) 213.

Braun, R. and Walterbos R. A. M. 1991, Astrophys. J., in press.

Brinks, E. 1981, Astron. Astrophys, 95, L1.

Brinks, E. and Bajaja, E. 1986 Astron. Astrophys, 169, 14.

Brinks, E., Braun, R. and Unger. S. W., 1989, in Structure and Dynamics of the Interstellar Medium, IAU Coll. 120, ed. G. Tenorio-Tagle, M. Moles, J. Melnick, Springer Verlag.

Casoli, F, Clausset, F., Viallefond, F., Combes, F. and Boulanger, F. 1991, in The Interstellar Medium in External Galaxies, NASA Conf. Publ. 3084, ed. D. J. Hollenbach and H. A. Thronson, 371.

Clark, B. G. 1965, Astrophys. J., 142, 1398.

Colgan, S. W. J., Salpeter, E. E. and Terzian, Y. 1988, Astrophys. J., 328, 275.

Courtèz, G., Petit, H., Sivan, J,-P., Dodonov, S., and Petit, M, 1988, Astron. Astrophys, 174, 28.

Cox, D. P. and Smith, B. W. 1974, Astrophys. J., 189, L105.

Davies, R. D., Elliott, K. H. and Meaburn, J. 1976, Mem. R. Astr. Soc., 81, 89.

Deul, E. and den Hartog, R. H. 1990, Astron. Astrophys, 229, 362.

Dickey, J. M., Salpeter, E. E. and Terzian, Y. 1978, A strophys. J. Suppl, 36, 77.

Field, G. B., Goldsmith, D. W. and Habing H. J. 1969, A strophys. J., 155. L149.

García-Burillo, S., Dahlem, M., and Guélin M. 1991, in The Interstellar Disk-Halo Connection in Galaxies, IAU Symp. 144, ed. J. B. G. M. Bloemen, (Kluwer Acad. Publ.) 299.

Heiles, C. 1979, Astrophys. J., 229, 533.

Heiles, C. 1984, Astrophys. J. Suppl, 55, 585.

Heiles, C. 1990, A strophys. J., 354, 483.

Heiles, C. 1991, in The Interstellar Disk-Halo Connection in Galaxies, IAU Symp. 144, ed. J. B. G. M. Bloemen, (Kluwer Acad. Publ.) 433.

Hunter, D. A. and Gallagher J. S. (1990), A strophys. J., 555, 999.

Kamphuis, J., Sancisi, R. and van der Hulst, J. M. 1991, Astron. Astrophys., 244, L29.

Kenney, J. D. P. 1990, in The Interstellar Medium in Galaxies, eds. H. A. Thronson and J. M. Shull, (Kluwer Aced. Publ.), 151.

Kulkarni, S. R. and Heiles C. 1988, in Galactic and Extragalactic Radio Astronomy, eds. G. L. Verschuur ans K. I. Kellermann (N.Y. Springer Verlag) 95.

Lasker, B. M. 1977, Astrophys. J., 212, 390.

Lasker, B. M. 1977, Pub. A. S. P., 91, 153.

Lockman, F. J. 1984, Astrophys. J., 283, 90.

Lockman, F. J. 1991, in The Interstellar Disk-Halo Connection in Galaries, IAU Symp. 144, ed. J. B. G. M. Bloemen, (Kluwer Acad. Publ.) 15.

Lord, D. L. and Kenney, J. D. P. 1991, Astroph. J., 381, 130. 
Meaburn, J. 1980, M.N.R.A.S., 192, 365.

Mathis, J. 1986, Astrophys. J., 301, 423.

Mac Low, M. and McCray, R., 1988, Astrophys. J., 324, 776.

Mac Low, M., McCray, R. and Norman, C. 1989, Astrophys. J., 337, 141.

McKee, C. F. and Ostriker, J. P. 1977, Lasker, B. M. 1977, Astrophys. J., $218,148$.

Norman, C. A. and Ikeuchi S. 1989, Astrophys. J., 345, 372.

Norman, C. A. 1991, in The Interstellar Disk-Halo Connection in Galaxies,

IAU Symp. 144, ed. J. B. G. M. Bloemen, (Kluwer Acad. Publ.) 337.

Payne H. E., Salpeter, E. E. and Terzian, Y. 1982, Astrophys. J. Suppl., 48, 199.

Payne H. E., Salpeter, E. E. and Terzian, Y. 1983, Astrophys. J., 272, 540.

Rand, R. J. and Kulkarni S. R. 1990, Astrophys. J., 349, L43.

Rand, R. J., Kulkarni, S. R. and Hester, J. J. 1990, A strophys. J., 352, L1.

Rand, R. J., Kulkarni, S. R. and Hester, J. J. 1991, Astrophys. J., in press.

Rand, R. J., Kulkarni, S. R. and Rice, W. 1991, Astrophys. J., in press.

Reynolds, R. J. 1984, Astrophys. J., 294, 256.

Reynolds, R. J. 1991, in The Interstellar Disk-Halo Connection in Galaxies,

IAU Symp. 144, ed. J. B. G. M. Bloemen, (Kluwer Acad. Publ.) 67.

Rice, W., Boulanger, F., Viallefond, F., Soiffer, B. T. and Freedman, W., L. 1990, Astrophys. J., $358,418$.

Savage, B. D. 1990, in The Interstellar Disk-Halo Connection in Galaxies,

IAU Symp. 144, ed. J. B. G. M. Bloemen, (Kluwer Acad. Publ.) 131.

Savage, B. D. and de Boer K. S. 1979, Astrophys. J., 230, L77.

Savage, B. 1991, in The Interstellar Disk-Halo Connection in Galaries,

IAU Symp. 144, ed. J. B. G. M. Bloemen, (Kluwer Acad. Publ.) 131.

Stark, A. A. 1979, Ph.D. Thesis, Princeton University.

Tacconi, L. J. and Xie, S. 1991, in The Interstellar Medium in External Galaxies,

NASA Conf. Publ. 3084, ed. D. J. Hollenbach and H. A. Thronson, 371.

Tenorio-Tagle, G. and Bodenheimer, P. 1988, Ann. Rev. Astron. Astrophys., 26, 145.

Tilanus R. P. J. and Allen R. J. Astrophys. J., 339, L57.

Young, J. S. 1990, in The Interstellar Medium in Galaxies, eds. H. A. Thronson and J. M. Shull, (Kluwer Aced. Publ.), 67.

Young J. S. and Knezek, P. 1989, Astrophys. J., 347, L55.

Young J. S. and Scoville, N. Z. 1982, Astrophys. J., 260, L11.

Young J. S. and Scoville, N. Z. 1991, Ann. Rev. Astron. Astrophys., 29, 581.

van der Hulst, J. M. and Sancisi, R. 1988, Astron. J., 95, 1354.

van der Hulst, J. M. and Kamphuis, J. 1991, in The Interstellar Disk-Halo Connection

in Galaxies, IAU Symp. 144, ed. J. B. G. M. Bloemen, (Kluwer Acad. Publ.) 201.

Wainscoat, R. J., de Jong, T. and Wesselius, P. R. 1987, Astron. Astrophys, 181, 22.

Walterbos, R. A. M. and Schwering, P. B. W. 1987, Astron. Astrophys, 180, 27.

Walterbos, R. A. M. 1990, in The Interstellar Disk-Halo Connection in Galaxies, IAU Symp. 144, ed. J. B. G. M. Bloemen, (Kluwer Acad. Publ.) 223. 